\title{
Intrinsic granularity in nanocrystalline boron-doped diamond films measured by scanning tunneling microscopy
}

\author{
B. L. Willems, ${ }^{1,2, *}$ V. H. Dao-,${ }^{3}$ J. Vanacken, ${ }^{1}$ L. F. Chibotaru, ${ }^{3}$ V. V. Moshchalkov, ${ }^{1}$ I. Guillamón, ${ }^{4}$ H. Suderow, \\ S. Vieira, ${ }^{4}$ S. D. Janssens, ${ }^{5}$ O. A. Williams, ${ }^{5,6}$ K. Haenen, ${ }^{5,6}$ and P. Wagner ${ }^{5,6}$ \\ ${ }^{1}$ Institute for Nanoscale Physics and Chemistry (INPAC), Nanoscale Superconductivity and Magnetism \& Pulsed Fields Group, \\ Katholieke Universiteit Leuven, Celestijnenlaan 200D, Leuven B-3001, Belgium \\ ${ }^{2}$ Facultad de Ciencias Físicas, Universidad Nacional Mayor de San Marcos, P.O. 14-0149, Lima 14, Peru \\ ${ }^{3}$ Institute for Nanoscale Physics and Chemistry (INPAC) and Division of Quantum and Physical Chemistry, University of Leuven, \\ Celestijnenlaan 200F, B-3001 Leuven, Belgium \\ ${ }^{4}$ Departamento de Física de la Materia Condensada, Instituto de Ciencia de Materiales Nicolás Cabrera, Laboratorio de Bajas \\ Temperaturas, Facultad de Ciencias, Universidad Autónoma de Madrid, 28049 Madrid, Spain \\ ${ }^{5}$ Institute for Materials Research (IMO), Hasselt University, BE-3590 Diepenbeek, Belgium \\ ${ }^{6}$ Division IMOMEC, IMEC vzw, Wetenschapspark 1, BE-3590 Diepenbeek, Belgium
}

(Received 19 January 2009; revised manuscript received 26 November 2009; published 21 December 2009)

\begin{abstract}
We report on low-temperature scanning tunneling microscopy/spectroscopy experiments performed on superconducting boron-doped nanocrystalline diamond (NCD) thin films prepared by chemical-vapor deposition methods. The most representative sample reveals the observed superconducting gap $(\Delta)$ highly modulated over a length scale on the order of $\sim 30 \mathrm{~nm}$, which is much shorter than the typical diamond grain size. The sample local and macroscopic behavior favors for the $\Delta$ modulation as being an intrinsic property of the NCD granules. On the other hand, $\Delta$ shows its temperature dependence $[\Delta(T)]$ consistent with the results obtained by Fominov and Feigel'man [Phys. Rev. B 63, 094518 (2001)], who studied theoretically the behavior of the superconducting gap of a BCS superconductor in contact with a normal layer by solving the one-dimensional Usadel equations on the superconducting side of the superconducting to normal interface.
\end{abstract}

DOI: $10.1103 /$ PhysRevB.80.224518

PACS number(s): 74.50.+r, 74.81.Bd, 74.78.Db

\section{INTRODUCTION}

Due to its outstanding electronic, mechanical, and thermal properties, diamond became a potential candidate for developing electronic devices which could meet the increasing demand ${ }^{1}$ for smallness, higher performance, and less power consumption and heat dissipation. Furthermore, the material rigidity and electrochemical robustness make it of interest for high-temperature electronic application such as diamond transistors $^{2}$ that could operate at $T>700 \mathrm{~K}$.

Besides of its potential technological applications, the fundamental properties of diamond constitute by themselves intriguing and fascinating research topics. For example, the evolution of the transport behavior on the boron dopant concentration might help understanding more general aspects such as the metal-insulator transition and its correlation with the appearance of a superconducting state. In 2004, the discovery of superconductivity in highly boron-doped diamond films has been reported, ${ }^{3}$ adding a new property to the already broad phenomena exhibited by these materials. Pure diamond is known to be a wide-gap insulator with a $5.5 \mathrm{eV}$ band gap and, for boron dopant concentrations of $n_{B}$ $\sim 10^{17} \mathrm{~cm}^{-3}$, an acceptor ground level is located at approximately $370 \mathrm{meV}$ above the host's valence band. ${ }^{4}$ So far, superconductivity has only been observed when the system is in the metallic regime, ${ }^{3,5-7}$ i.e., when the boron concentration $\left(n_{B}\right)$ exceeds a critical value $n_{C}$. For the case of diamond films prepared by chemical-vapor deposition (CVD) methods, the experimental values for $n_{C}$ have been found to lie between ${ }^{5,6} 2 \times 10^{20}$ and $4.5 \times 10^{20} \mathrm{~cm}^{-3}$. On the other hand, single-crystalline boron-doped diamond has revealed its critical temperature $\left(T_{C}\right)$ to depend on $n_{B}$ as ${ }^{5} T_{C} \sim\left(n_{B} / n_{C}\right.$ $-1)^{1 / 2}$. So far, the highest $T_{C}$ obtained for boron-doped diamond $^{8}$ is $11.4 \mathrm{~K}$.

CVD boron-doped nanocrystalline diamond (NCD) films have revealed their surface as composed of crystalline nanograins, displaying well-defined three-dimensional geometrical shapes ${ }^{9}$ such as pyramids and hexagons. In these, planes and vertexes are identified under scanning electron microscopy (SEM) imaging, each plane corresponding to a singlecrystal growth. ${ }^{10}$ Both superconductivity and the structural properties of these materials offer an appropriate scenario for studying regularly shaped superconductors with sizes beyond the achievable by standard lithographic techniques. Moreover, the grains are potentially interesting systems for being investigated with local techniques such as scanning tunneling microscopy (STM) and scanning tunneling spectroscopy (STS).

Among the few reports concerning STM and STS measurements on superconducting boron-doped diamond films, the work done by Sacepe et al. ${ }^{11}$ is particularly noteworthy. In this, single-crystalline diamond films have revealed a homogeneous superconducting gap $\Delta$ under local spectroscopic measurements. ${ }^{11}$ Furthermore, the temperature evolution of $\Delta$ is in agreement with the dependence predicted by the BCS theory. ${ }^{11}$ Recently, Ekimov et al. ${ }^{12}$ were able to measure the isotope effect of the carbon on the $T_{C}$. These two independent observations ${ }^{11,12}$ strongly supports the phonon-BCS mechanism for the superconductivity in diamond.

Here, we report on STM and STS studies performed on superconducting boron-doped NCD films prepared by CVD methods. These were initially aimed to explore locally the 
evolution of the superconducting properties along the planes and vertexes revealed by the granules in order to study confinement effects. It was expected to find the superconducting order parameter to be modulated due to the granularity, with the characteristic period coinciding with an average grain size. Contrary to that, we have found a pronounced intrinsic intragrain modulation of $\Delta$ by revealing the $\Delta$ to vary typically over $30 \mathrm{~nm}$, a distance much smaller than the characteristic grain size. Moreover, coexisting normal and superconducting regions were resolved inside the granules as well as in between them. A comparative analysis of the macroscopic and local properties of our samples supports the intragrain modulation of $\Delta$ as being an intrinsic property of the NCD granules. It will be shown that our results are in agreement with the recent observation of modulated local density of states (LDOS) on surfaces of boron-doped diamond films. ${ }^{13}$

On the other hand, the superconducting gap $\Delta$, extracted from the $I-V$ curves measured at a fixed point and at different temperatures, shows its temperature dependence $[\Delta(T)]$ different from that predicted by the BCS theory. The $\Delta(T)$ is significantly affected between 2 and $1 \mathrm{~K}$, below which it saturates. Supported by numerical solutions of the onedimensional (1D) Usadel equations, our experimental data give direct evidence that proximity effects are responsible for the departure from the conventional BCS behavior. As far as we know, the temperature evolution of the superconducting gap in the presence of proximity effects has not been extensively studied experimentally in superconducting diamond, if not at all. On the contrary, research has been focused mainly on the spatial evolution of $\Delta$ with respect to a superconductor/normal (SN) interface instead. ${ }^{14-16}$ However, the $\Delta(T)$ dependence in the proximity of a $\mathrm{SN}$ interface has been treated theoretically by Fominov and Feigel'man. ${ }^{17}$

\section{EXPERIMENT}

The boron-doped NCD films were prepared by plasmaassisted CVD techniques. Diamond layers were grown onto $\mathrm{Si}$ substrates in a $\mathrm{H}_{2} / \mathrm{CH}_{4}$ plasma, with methane concentrations below $3 \%$, at $700{ }^{\circ} \mathrm{C}$ and under pressures of 30 Torr. Boron doping was achieved by injecting trimethylboron $\left[\mathrm{B}\left(\mathrm{CH}_{3}\right)_{3}\right]$ gas during whole growth process with a $\mathrm{B}\left(\mathrm{CH}_{3}\right)_{3} / \mathrm{CH}_{4}$ ratio of $6500 \mathrm{ppm}$. Different growth times were used for each sample. Further details are reported elsewhere. ${ }^{9,18}$

To study the samples macroscopic properties, electrical transport and magnetic measurements were performed. The electrical transport properties were probed by using a fourterminal configuration with the contacts silver pasted to the corners of the $1 \times 1 \mathrm{~cm}^{2}$ samples. Current is caused to flow along one edge of the sample while the voltage was measured between the extremities of the opposite edge. We have found that the resistance measured by using this configuration showed almost no difference with the obtained when a transport bridge was patterned on one of the samples. The latter evidences the absence of important current redistribution processes in the whole temperature and field range, and hence the sample's extrinsic (resistance) and intrinsic (resis- tivity) parameters only differ by a constant. The temperatures between 272 and $1.5 \mathrm{~K}$ were obtained in an Oxford ${ }^{4} \mathrm{He}$ cryostat. This setup is provided with a $12 \mathrm{~T}$ dc magnet coil and a Lakeshore temperature controller. Four-point resistivity measurements were performed with a nanovoltmeter (Hewlett Packard 34420A) and a programmable current source (Keithley 220). To eliminate thermal or offset voltages while using the nanovoltmeter, the measured voltage is averaged over both positive and negative polarities of the injected current. Typically a current of $10 \mu \mathrm{A}$ is used.

The magnetic properties of some of the samples were additionally probed by measuring the temperature dependence of their magnetic moments, $M(T)$. For these, a "magnetic properties measurement system" (MPMS-XL) device from Quantum Design was used. The basic element of operation is an rf-SQUID (superconducting quantum interference device) (Ref. 19) which act as a very accurate flux-voltage converter. Magnetic moments as small as $10^{-8}$ emu can be measured with deviations of $10^{-9}$ emu. Typically, the measurements were conducted at $1.5 \mathrm{~cm}$ scan length with a reported field homogeneity of $0.005 \%$. Moreover, parasitic magnetic signals were reduced to the minimum by carrying out a demagnetization procedure prior to a measurement. This setup has a stable temperature range between 4 and $300 \mathrm{~K}$.

Low-temperature STM/STS measurements were performed on three of the superconducting NCD samples in a home-build STM setup installed in a dilution refrigerator. The system is mechanically decoupled of external vibrations and has a resolution in energy of ${ }^{20,21} 15 \mu \mathrm{eV}$. Furthermore, this setup has been thoroughly tested on several samples in the past. ${ }^{20,21}$ For the present experiment, a sharpened golden wire was used as a scanning tip. The microscope scanning window is $2.2 \times 2.2 \mu^{2}$ in the temperature range between 2.7 and $0.01 \mathrm{~K}$. dc magnetic fields were applied perpendicular to the sample surface. Topographic STM images were taken at a constant tunneling current. Local spectroscopic information was obtained by deriving the tunneling $I-V$ characteristics $[\sigma(V)=d I / d V(V)]$, ranging from voltages above the superconducting gap. STS maps, such as the one shown in Fig. 2(b), were then constructed by subtracting the zerobias conductance, $\sigma(0)$, from the constant normal background, ${ }^{22} \sigma_{\mathrm{N}}$. The brighter pixels in Fig. 2(b) correspond to larger $\sigma_{\mathrm{N}}-\sigma(0)$ while the dark spots indicate $\sigma_{\mathrm{N}}$ $-\sigma(0)=0$, i.e., they are normal regions. We have to note here that the $I-V$ curves taken on these samples were highly reproducible without any loss of the tunneling current, no matter whether the regions scanned were semiconducting or metallic.

\section{RESULTS AND DISCUSSION}

\section{A. Macroscopic properties}

All superconducting samples show similar characteristics for their resistance versus temperature dependencies $[R(T)]$ : (1) a broad transition toward a state of zero resistance, a characteristic for granular superconductivity, ${ }^{7,23}$ (2) the values for their $T_{C}$ are below $4 \mathrm{~K}$ when taking the half value of the normal resistance criterion, and (3) the $R(T)$ curves measured under 0 and $12 \mathrm{~T}$ magnetic fields split typically at 

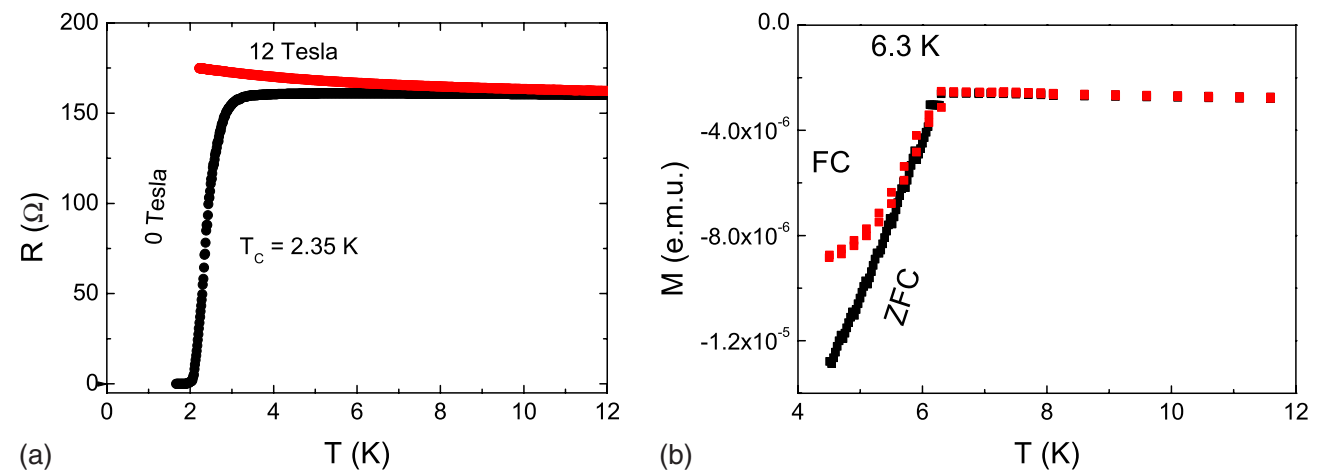

FIG. 1. (Color online) (a) A typical resistance versus temperature dependence of a superconducting NCD sample. The broad superconducting transition is revealed by the splitting of the $R(T)$ curves measured under 0 and $12 \mathrm{~T}$ fields at temperatures above $8 \mathrm{~K}$. A $T_{C}$ $=2.35 \mathrm{~K}$ has been obtained for this particular sample when taking the half value of its normal-state resistance. (b) The corresponding zero-field-cooling and field-cooling magnetic moment versus temperature dependence measured in a SQUID magnetometer. The onset of the increase in the sample's diamagnetic signal occurs at $6.3 \mathrm{~K}$ when a small field of $20 \mathrm{mT}$ is applied.

temperatures above $8 \mathrm{~K}$ [Fig. 1(a)]. On the other hand, the samples reveal an increase in their diamagnetic response at temperatures well above $T_{C}$ when measured in a SQUID magnetometer. This can be seen in Fig. 1(b), in which the temperature dependence of the magnetic moment of a representative sample is shown. Although the latter has a $T_{C}$ $=2.35 \mathrm{~K}$ [Fig. 1(a)], the onset of the increase in the diamagnetic response occurs at $6.3 \mathrm{~K}$ for an applied field of $20 \mathrm{mT}$. Both results, namely, those obtained from magnetic and transport measurements, show that the superconducting transition of the samples starts at temperatures two to three times higher than $T_{C}$. The latter behavior can be explained by an inhomogeneous doping of the sample. First, highly doped regions become superconducting around $6.3 \mathrm{~K}$ and their presence is detected by magnetic measurements. Next, a state of zero resistance is achieved below $2 \mathrm{~K}$ once percolating paths of Josephson-coupled regions are provided. ${ }^{24}$

\section{B. Local properties}

\section{Intrinsic granularity}

Table I summarizes the structural and superconducting properties of the three samples, labeled as s2, s3, and s5, whose low-temperature local properties were probed with

TABLE I. Superconducting and structural parameters of the samples studied. Grain sizes and film thicknesses were inferred from SEM and cross-sectional SEM imaging, respectively. Critical temperatures and fields, $T_{C}$ and $H_{C 2}$, respectively, were extracted from the $R(T)$ curves at the values corresponding to the half of the maximum resistance. The zero-temperature Ginzburg-Landau coherence length, $\xi(0)$, was obtained from the $H_{C 2}(0)$ value extracted from the slopes of the $H_{C 2}(T)$ dependencies.

\begin{tabular}{lccccc}
\hline \hline Sample & $\begin{array}{c}\text { Grain size } \\
(\mathrm{nm})\end{array}$ & $\begin{array}{c}\text { Film thickness } \\
(\mathrm{nm})\end{array}$ & $\begin{array}{c}T_{C} \\
(\mathrm{~K})\end{array}$ & $\begin{array}{c}H_{C 2}(0) \\
(\mathrm{T})\end{array}$ & $\begin{array}{c}\xi(0) \\
(\mathrm{nm})\end{array}$ \\
\hline s2 & 50 & 85 & 1.6 & 2.7 & 11 \\
s3 & 300 & 200 & 2.78 & 4.3 & 8.7 \\
s5 & 1000 & 800 & 3.1 & 4.1 & 8.9 \\
\hline \hline
\end{tabular}

STM. It has been found that $\Delta$ could be only observed in two of the samples while it was totally absent in the scanned area of s2. Moreover, in contrast to s3 and s5, the voltages required for producing a tunneling current in the scanned areas on sample s2 were higher than $2 \mathrm{~V}$, evidencing the semiconducting character of those regions. On the other hand, $\Delta$ could be only measured on very small spots over the surface of sample s5 while it was better resolved on s3. The latter revealed larger regions with homogeneous $\Delta$ and hence enabled to study its temperature dependence since the anchoring of the STM tip to a particular point is subjected to space fluctuations caused by thermal noise. ${ }^{11,25}$ Therefore, in what follows we will present and discuss in detail the data obtained on this particular sample which has a typical thickness of $200 \mathrm{~nm}$ and a lateral grain size of $300 \mathrm{~nm}$, inferred from both STM and SEM imagings.

Figure 2(a) shows the topography corresponding to a $560 \times 560 \mathrm{~nm}^{2}$ STM scanned region and Fig. 2(b) its corresponding STS map obtained from $I-V$ measurements performed at $0.1 \mathrm{~K}$. As mentioned before, the latter was constructed by subtracting the zero-bias conductance from the normal background, $\sigma_{\mathrm{N}}-\sigma(0)$. A comparison of both maps shows that the nucleation of superconductivity is highly in-

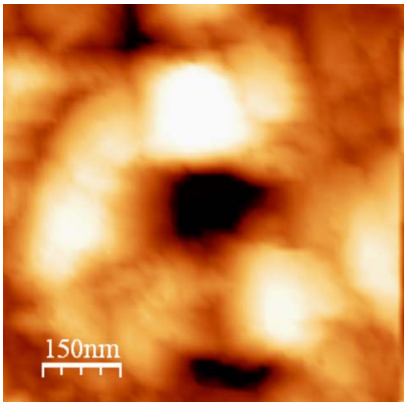

(a)

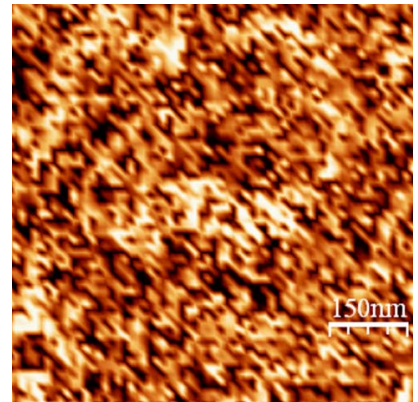

(b)
FIG. 2. (Color online) (a) A $560 \times 560 \mathrm{~nm}^{2}$ STM scanned area and (b) the STS map constructed from the $I$ - $V$ curves measured on $64 \times 64$ equidistanced points on the region displayed in (a), at a temperature of $0.1 \mathrm{~K}$. The brighter pixels in (b) indicate a larger $\sigma_{\mathrm{N}}-\sigma(0)$ while the dark spots correspond to normal regions. 
homogeneous over the film surface. Furthermore, crosssectional analysis of the STS maps shows that $\Delta$ changes typically over $\sim 30 \mathrm{~nm}$, a considerable smaller length scale compared to the NCD grain size. This indicates clearly the presence of the intragrain modulation of $\Delta$.

As is known, the quality of a STM measurement is subjected to many parameters, being surface contamination a fundamental issue. The latter often prevents stable and reproducible tunnel junctions and the investigation of intrinsic properties. ${ }^{25}$ It is important here to remark the high reproducibility of the tunnel currents in our samples, no matter how fast the measurements were performed. Slow and fast scans gave exactly the same results even in the case of sample s2, in which the LDOS of a semiconductor was measured. On the other hand, similar observations as those shown in Fig. 2(b) were reported for a superconducting TiN thin film, ${ }^{16}$ which reveals its surface as consisting of normal and superconducting regions under STM. An important proof for the inhomogeneities as being intrinsic to the sample is attributed to the smooth transition from the superconducting to the normal regions, with no change in the noise of the STS data. ${ }^{16}$ Moreover, at low temperatures, surface contamination due to adsorbates are claimed to be detectable with STM by the observation of bumps in the topographic images and by measuring the LDOS of an insulator. ${ }^{16}$ The latter were totally absent in the scanned areas of sample s3; even more, no important changes were observed in the noise of the STS data when transiting from a superconducting to a normal region inside the grains.

Another possible cause for the inhomogeneous $\Delta$ could be attributed to the composition of the boron-doped diamond surface. In particular, the boundaries of heavily boron-doped NCD grains are expected to be constituted of $s p^{2}$ graphite layers with boron-rich $\mathrm{B}_{x} \mathrm{C}_{1-x}$ phases. . $^{9,18,26}$ These normal layers could influence the measurement of $\Delta$ by proximity effects. In fact, as will be seen below, the temperature dependence of the conductance is well reproduced theoretically by considering the diamond grains covered with a thin normal layer on top. However, since the superconducting gap inside the normal layer is expected to evolve with distance as ${ }^{27}$ $\Delta(x) \propto \exp (-x / \xi)$, where $\xi$ is the superconducting coherence length $(\sim 10 \mathrm{~nm}$, see Table I) and $x$ is taken from the SN interface; the change in $\Delta$ shown in Fig. 2(b) would imply that the normal layer thickness varies several times $\xi$ over a length scale on the order of $30 \mathrm{~nm}$.

The above analysis suggests that the observed $\Delta$ modulation is at least an intrinsic property of the NCD grain surfaces. An additional support for the latter comes from the correlation existing between the $H_{C 2}(T)$ dependencies obtained from STS and electrical transport measurements (Fig. $6)$. As will be discussed in the last section, the correspondence observed is consistent with regarding the sizes of the superconducting grains below $r<37 \mathrm{~nm}$, on the order of the modulation length inferred from Fig. 2(b).

The $\Delta$ nanomodulation observed in sample s 3 is in agreement with the rencent results reported by Altfeder et al., ${ }^{13}$ who studied the surfaces of boron-doped diamond films by performing STM experiments. It has been found that these surfaces reveal spatially ordered magic-sized nanocrystals by showing an arrangement of the LDOS in parallelograms with base and height sizes equal to 8.3 and $10.5 \mathrm{~nm}$, respectively. ${ }^{13}$ Such arrangement is completely absent in pure nondoped diamond samples and is claimed to be caused by the Fermi-sea-induced quantum electronic growth mechanism. ${ }^{13}$ According to the latter, the electron de Broglie wavelength controls the geometry of the nanostructures, establishing a preferred size for them. ${ }^{13}$ Moreover, as can be inferred from Figs. 2(a) and 2(b) of Ref. 13, the nanocrystals also display a preferred orientation. Remarkably, the superconducting and normal regions observed in the STS map of our sample [Fig. 2(b)] show their characteristics similar to the diamond nanocrystals of Altfeder et al., ${ }^{13}$ namely, a preferred orientation can be identified and many of them display parallelogramlike shapes. Although there is an important difference in the modulation length (30 nm versus $10.5 \mathrm{~nm}$ ), which can be attributed to many factors such as sample surface quality, difference in the values for $n_{B}$, inhomogeneous boron incorporation, etc; our results essentially confirm those of Altfeder et al. ${ }^{13}$

Most probably, the change in $\Delta$ is caused by the inhomogeneous boron incorporation by the diamond grains. The latter might affect the $T_{C}$ locally since it has been found to vary with $n_{B}$ as $T_{C} \sim\left(n_{B} / n_{C}-1\right)^{1 / 2}$. Taking into account that the NCD grains are formed of single-crystalline facets with different orientations, ${ }^{10}$ a correlation between Figs. 2(a) and 2(b) suggests that these facets also have an inhomogeneous distribution of $n_{B}$. Here, it is important to discuss the following: recent STM studies ${ }^{28}$ on boron-doped single-crystalline films show that although having the samples an inhomogeneous boron incorporation, revealed by the difference in the spectra measured over different points at $T \sim T_{C}$, the superconducting $\Delta$ was spatially homogeneous at $T \ll T_{C}$. Since at $0.1 \mathrm{~K} \ll T_{C} \Delta$ also varies on the single-crystalline facets of the NCD grains of sample s3, the difference between our results and those reported in Ref. 28 leaves the possibility open for other mechanisms as causing the $\Delta$ modulation.

It is worth mentioning that strong intrinsic $\Delta$ nanomodulation in boron-doped diamond reminds the similar observations made on $\mathrm{Bi}_{2} \mathrm{Sr}_{2} \mathrm{CaCu}_{2} \mathrm{O}_{8+\delta}$ (B-2212) high- $T_{C}$ superconductors, ${ }^{29,30}$ where this $\Delta$ modulation is attributed to the quasiparticle scattering between high joint density of states regions of the $\vec{k}$ space. ${ }^{30,31}$ Similar effects could take place in heavily boron-doped diamond, where the important admixture of the anisotropic $L$ bands is present at the Fermi level. ${ }^{32,33}$ Under these conditions, contrary to the pure $\Gamma$-like Fermi surface, a few $\vec{k}$ vectors with a much higher statistical weight can also be found to interfere strongly within a grain $^{34}$ thus leading to a pronounced intrinsic intragrain nanomodulation of $\Delta$. The possibility for the boron-doped diamond electronic band structure as responsible not only for the surface LDOS modulation, but also for the $\Delta$ modulation, is currently under study.

\section{Temperature dependence of $\Delta$}

Figure 3 shows the normalized conductance $\sigma_{\text {norm }}$ versus the bias-voltage dependencies, which were extracted from the $I-V$ curves measured at different temperatures and on a fixed point. Here, the normalized conductance is defined as $\sigma_{\text {norm }}=\sigma(V) / \sigma_{\mathrm{N}}$. Contrary to the case of single-crystalline 


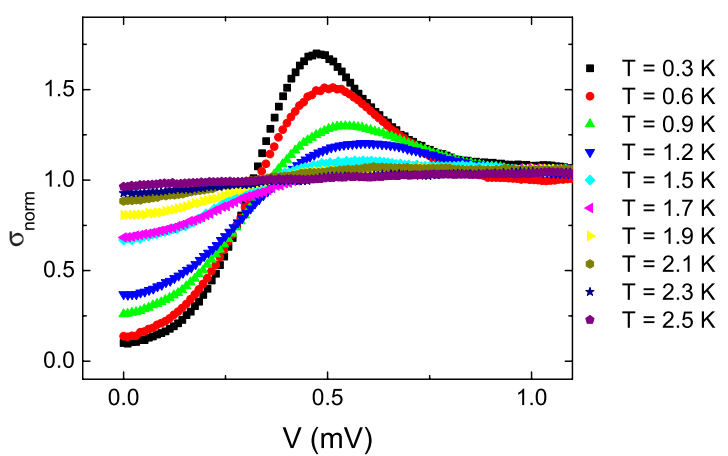

FIG. 3. (Color online) The normalized conductance $\sigma_{\text {norm }}$, obtained by deriving the measured $I(V)$ characteristics at a fixed point and at different temperatures versus the bias voltage (units in millivolts).

boron-doped diamond, where $\sigma(V)$ has a BCS U-like shape with $^{11} \sigma(0)=0 ; \sigma_{\text {norm }}$ is nonzero at zero bias and shows a V-like shape even at the lowest temperatures. Such a change in shape has been reported for systems having SN proximity junctions $^{14,15}$ and is attributed to the effect of diffusive Cooper pairs on the density of states of the normal part. In these SN contacts, $\sigma_{\mathrm{N}}-\sigma(0) \rightarrow 0$ when moving deeper into the normal part.

The coexistence of normal and superconducting regions on the sample surface [Fig. 2(b)] as well as the possibility for the existence of thin normal layers separating superconducting crystals from the surface, motivates the importance of the consideration of proximity effects when attempting to formulate a quantitative interpretation of the results. Systems dealing with such effects have been well reproduced by solving the $1 D$ Usadel diffusion equation. ${ }^{35}$ In the present case, one BCS superconducting grain (S) and its normal outer layer (N) are modeled as a SN bilayer of respective thicknesses $L_{\mathrm{S}}$ and $L_{\mathrm{N}}$. The density of states is calculated within the quasiclassical real-time Green's-function formalism. In the dirty limit, the Usadel equation ${ }^{35}$ for the retarded Green's functions $G=\cos \theta$ and $F=-i \sin \theta$ at equilibrium in zero field reads

$$
\frac{D}{2} \partial_{x}^{2} \theta+\left(i E-\Gamma_{i n}\right) \sin \theta+\Delta \cos \theta=0
$$

where $D$ is the diffusivity, $\Gamma_{\text {in }}$ is the inelastic-scattering rate, and $\theta=\theta(x, E)$ is a complex function of position $x$ and energy $E$. We take $\hbar=k_{B}=1$, where $k_{B}$ is the Boltzmann's constant. This equation is self-consistently solved by a numerical relaxation method with the equation for the order parameter,

$$
\Delta=\lambda \int_{0}^{\omega_{D}} d E \mathfrak{I}(\sin \theta) \tanh \left(\frac{E}{2 T}\right),
$$

where $\lambda$ is the superconducting pairing strength and $\omega_{D}$ is the cutoff frequency which is on the order of the Debye frequency. These parameters define the critical temperature $T_{C 0}=2 \gamma^{\prime} \omega_{D} e^{-1 / \lambda} / \pi$ and the zero-temperature gap value $\Delta_{0}$ $=\pi T_{C 0} / \gamma^{\prime}$ (with $\gamma^{\prime} \approx 1781$ ) of the bulk system in the clean limit. In the normal layer $\lambda$ and $\Delta$ are zero but a finite $F$ is induced by the proximity of the superconductor. The Usadel

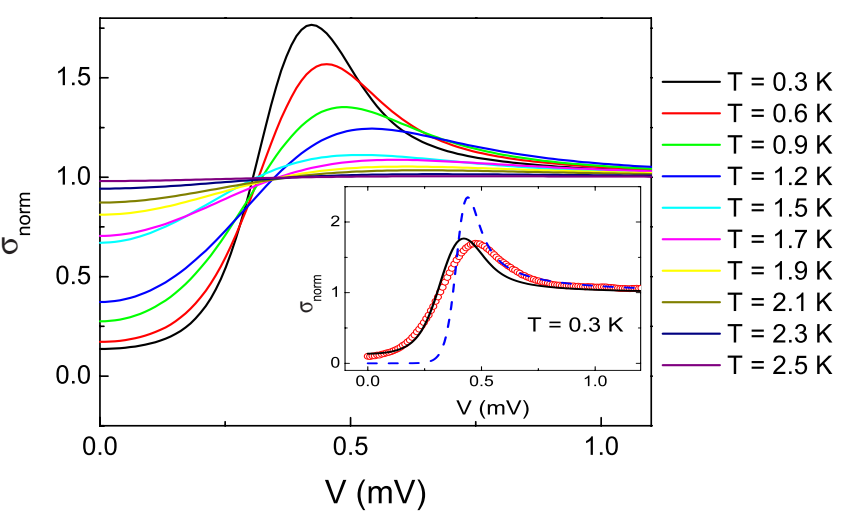

FIG. 4. (Color online) Fittings of the experimental data plotted in Fig. 3. (Inset) The fit performed on the $0.3 \mathrm{~K}$ experimental data (circles) by solving the Usadel (solid line) equations. As a comparison, the result obtained for the case in which the normal layer is absent is also shown (dashed line). This corresponds to the pure BCS situation.

equation is supplemented by the boundary conditions $\partial_{x} \theta(0)=0$ and $\partial_{x} \theta\left(L_{\mathrm{S}}+L_{\mathrm{N}}\right)=0$ at the extremities. ${ }^{36}$ At the SN interface $\sigma_{\mathrm{S}} \partial_{x} \theta\left(L_{\mathrm{S}}^{-}\right)=\sigma_{\mathrm{N}} \partial_{x} \theta\left(L_{\mathrm{S}}^{+}\right)=\sin \left[\theta\left(L_{\mathrm{S}}^{+}\right)-\theta\left(L_{\mathrm{S}}^{-}\right)\right] / R_{\text {int }}$ with the normal-state conductivity in the $\mathrm{S}(\mathrm{N})$ layer $\sigma_{\mathrm{S}(\mathrm{N})}$ $=2 e^{2} N(0) D_{\mathrm{S}(\mathrm{N})}$ and the resistance at the interface $R_{\text {int }}$. The obtained LDOS $N(E)=N(0) \Re(\cos \theta)$ at $x_{p}=L_{\mathrm{S}}+L_{\mathrm{N}}$ is convoluted with the derivative of the Fermi-Dirac distribution at the temperature of the probe $T_{p}$ in order to compare it with the experimental $d I / d V$. Variations in $D_{\mathrm{N}}, \sigma_{\mathrm{N}}, R_{i n t}$, and $L_{\mathrm{N}}$ have similar effects on the LDOS at $x_{p}$. Because of the uncertainty on the interface quality, we then set $D_{\mathrm{N}} / D_{\mathrm{S}}$ $=\sigma_{\mathrm{N}} / \sigma_{\mathrm{S}}=1, R_{\text {int }}=0$ and use the normal layer thickness as an effective parameter. On the other hand, $\Gamma_{i n}$ depends linearly on the temperature, a typical dependence for onedimensional diffusive processes. ${ }^{37}$

The fits of the experimental data presented in Fig. 3 are plotted in Fig. 4, showing a good agreement with the experiment. These fits were obtained for $T_{C 0}=3.8 \mathrm{~K}$, a value that is consistent with previous results from magnetic and electrical transport measurements where the onset of the superconducting transition is observed above $6 \mathrm{~K}$ and the critical temperature, taken at the half value of the maximum resistance, is $T_{C}=2.78 \mathrm{~K}$. For all of the fits, the values used for the probe temperature $T_{p}$ were the same as those of the sample. This is consistent with the vast number of tests performed on the setup used, where it has been observed that above $0.15 \mathrm{~K}$ there exist almost no difference between the temperatures of the sample and the tip. ${ }^{20}$ In the inset of Fig. 4 , the $\sigma_{\text {norm }}$ corresponding to $T=0.3 \mathrm{~K}$ is plotted together with the fit found by using the 1D Usadel equation (solid line). For comparison, the $\sigma_{\text {norm }}$ obtained in the absence of proximity effects (i.e., when $L_{N}=0$ ) is also shown (dashed line) and corresponds to the BCS situation. The good agreement between theory and experiment, as well as the observation of similar features in the SN proximity junctions, ${ }^{14,15}$ confirms that the measured spectra are indeed affected by proximity effects.

As a consequence, $\Delta(T)$ shows to depart from the classical BCS dependence (solid lines in Fig. 5), tending to vary 


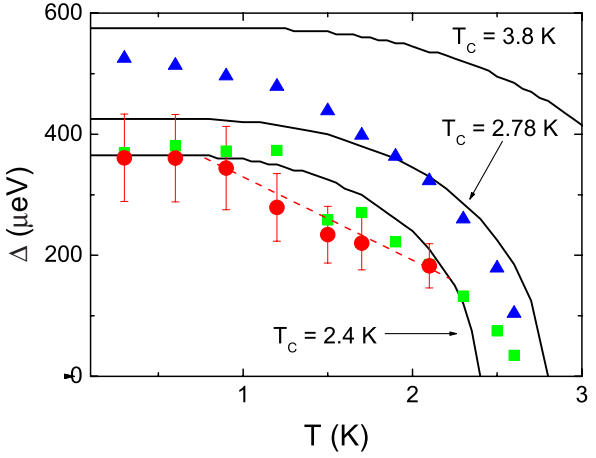

FIG. 5. (Color online) The (red) dots are the experimentally obtained $\Delta$ (with $20 \%$ error bars), corresponding to the voltage where $d \sigma / d V$ is maximum. The (green) squares and (blue) triangles are the calculated $\Delta$ by solving the Eq. (2) at $x=L_{S}$ and $x=0$, respectively. The solid (black) lines are the expected BCS dependence for $T_{C}=2.4 \mathrm{~K}, T_{C}=2.78 \mathrm{~K}$, and $T_{C}=3.8 \mathrm{~K}$, respectively. A $T_{C}$ $=2.78 \mathrm{~K}$ was obtained from transport measurements by taking the half value of the maximum resistance criterion. $T_{C}=2.4 \mathrm{~K}$ is the temperature above which $\sigma_{\text {norm }}(V)$ of Fig. 3 is constant. In addition, a dashed straight line is shown as a guide to the eyes.

linearly upon lowering the temperature between 2 and $1 \mathrm{~K}$, below which it evolves toward saturation. This is shown in Fig. 5 where the experimental $\Delta(T)$ (dots), taken as the voltage where $d \sigma / d V$ is maximum, ${ }^{38}$ are plotted together with the calculated $\Delta(T)$ at $x=L_{\mathrm{S}}$ (squares). The bars correspond to an error of $20 \%$ in the data acquisition and are shown in order to make the difference clear between the temperature dependence of $\Delta$ and the behavior predicted by the BCS theory for a $T_{C}=2.4 \mathrm{~K}$, temperature above which $\sigma_{\text {norm }}(V)$ is constant (see Fig. 3 ). The theoretical $\Delta$, obtained by solving Eq. (2) when fitting the data plotted in Fig. 3, shows the values close to the experimental data and reproduces the temperature dependence observed. Furthermore, the evolution of the $\Delta$ deep inside the superconductor, i.e, at $x=0$ (triangles), also shows to be affected by the normal layer. This can be seen by the significant departure showed from the BCS dependence (solid line).

The aforementioned behaviors are consistent with the results obtained by Fominov and Feigel'man, ${ }^{17}$ who studied theoretically the temperature evolution of the $\Delta$ of a BCS superconductor in contact with a normal layer, by solving the 1D Usadel equations on the superconducting side of the SN interface. Hence, our experimental data show that proximity effects not only alter the $\sigma(V)$ shape but also the temperature dependence of a BCS $\Delta$ when measured through a normal layer and both features are well reproduced by the solution of the 1D Usadel equation. It is important to remark that these results do not contradict those reported in Refs. 11 and 12. Here, the temperature dependence of $\Delta$ is that of a BCS $\Delta$ measured through a normal layer.

\section{3. $H_{C 2}(T)$ from local measurements}

Finally, boron-doped diamond films in general have revealed high values for their critical magnetic field ${ }^{3,39}\left(H_{C 2}\right)$. Such values are, however, not expected for a weak-coupled low- $T_{C}$ BCS superconductor and must be caused by confine-

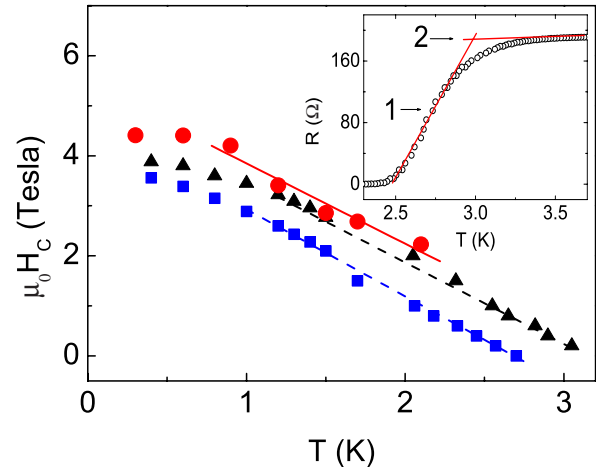

FIG. 6. (Color online) Correlation between the $H_{C 2}$ obtained by applying the local $\Delta(T)$ to the Clogston's formula (red circles) and the $H_{C 2}$ obtained from magnetoresistance measurements under the criteria shown in the inset: criteria 1 (blue squares) and 2 (black triangles).

ment effects. In particular, it has been found that when reducing the size of a superconducting grain below its bulk magnetic penetration depth, $H_{C 2}$ can have values even orders of magnitude higher than those for the bulk sample (e.g., Li et al. ${ }^{40}$ and references therein). This can be understood by noticing that the partial or complete field penetration inside such a small superconductor is accompanied with a reduction in the system's Meissner free energy. ${ }^{41}$ Clogston $^{41}$ argued that even in the absence of the Meissner effect, a limit is imposed by the normal state on the critical field. Moreover, the zero-temperature critical field of a BCS superconductor with a paramagnetic normal state (e.g., a metal) is obtained by $^{41}$

$$
\mu_{0} H_{C 2}(0)=\frac{\Delta(0)}{\mu_{B} \sqrt{2}},
$$

where $\mu_{B}$ is the Bohr magneton. Although the (paramagnetic pair breaking) Clogston's formula has been derived for a BCS superconductor at zero temperature and with a paramagnetic normal state, ${ }^{41}$ it correlates well with the temperature evolution of the $H_{C 2}(T)$ obtained from electrical transport measurements by inserting the values of $\Delta(T)$ in Eq. (3). This is shown in Fig. 6 where the calculated $H_{C 2}(T)$ (dots) is plotted together with the $H_{C 2}(T)$ extracted from macroscopic transport measurements (squares and triangles). The squares correspond to the $H_{C 2}(T)$ obtained by using the half value of the normal resistance criterion (shown as 1 in the inset) and the triangles by taking the points at which the constant normal resistance intersects the linear part of the transition (shown as 2 in the inset). Close values for $H_{C 2}(0)$ and $d H_{C 2}(T) / d T$ (between 4.65 and $5.97 \mathrm{~T}$, and 1.6 and $1.83 \mathrm{~T} / \mathrm{K}$, respectively) were obtained from the linear fitting at $T$ $>1 \mathrm{~K}$. The consistency of the local and macroscopic $H_{C 2}(T)$ dependencies is not fortuitous and can be explained by the following: the magnetic field required for breaking a Cooper pair by diamagnetic effects in a spherical grain of radius $r$ is given by ${ }^{42}$ 


$$
H_{o r b} \approx \frac{\Phi_{0}}{r^{2} \sqrt{g_{d i m}}} \mathrm{~T},
$$

where $\Phi_{0}$ is the quantum flux, $g_{d i m}=E_{T h} / d$ is the dimensionless conductance, $E_{T h}$ and $d$ are the grain Thouless energy and the mean energy-level spacing, respectively. In the freeelectron approximation $d$ is given by ${ }^{42}$

$$
d=\frac{1.5}{k_{F} \mathrm{Vol}} \mathrm{eV} \mathrm{nm}^{2},
$$

where $k_{F}$ is the Fermi vector and $\mathrm{Vol} \sim r^{3}$ is the grain volume. On the other hand, the expression for $E_{T h}$ in the diffusive regime reads ${ }^{42}$

$$
E_{T h}=\frac{0.25 k_{F} \ell}{r^{2}} \mathrm{meV} \mathrm{nm}{ }^{2},
$$

where $\ell$ is the transport mean-free path. By taking the values for ${ }^{11} \ell=1.3 \mathrm{~nm}$ and the $k_{F}$ for a heavily boron-doped diamond $^{33}$ between $^{43} 1.1$ and $2.7 \mathrm{~nm}^{-1}$; a $4.65<H_{\text {orb }}$ $<5.97 \mathrm{~T}$ corresponds to a grain radius between $37<r$ $<59 \mathrm{~nm}$. In other words, for grain sizes less than $37 \mathrm{~nm}$, paramagnetic pair breaking is expected. Hence, the high- $H_{C 2}$ values are consistent with the characteristic length of $\Delta$ found from the STS experiments [Fig. 2(b)].

\section{CONCLUSIONS}

In conclusion, we have performed STM and STS measurements on nanocrystalline boron-doped CVD diamond thin films at dilution fridge temperatures. In addition, the samples macroscopic properties were probed by performing electrical transport and magnetic measurements. The most representative sample reveals a strongly modulated $\Delta$, varying over the distances much shorter than the typical size of the NCD grains. Macroscopic and local experimental results strongly supports the observed pronounced intragrain nanomodulation of $\Delta$ as being an intrinsic property of the NCD granules and is most likely caused by an inhomogeneous boron doping. However, the possible implication of the boron-doped diamond electronic band structure on the modulation of $\Delta$ cannot be fully ruled out. On the other hand, $\Delta$ shows its temperature dependence consistent with the results obtained by Fominov and Feigel'man, ${ }^{17}$ who studied theoretically the behavior of the superconducting gap of a BCS superconductor in contact with a normal layer by solving the 1D Usadel equations on the superconducting side of the SN interface.

\section{ACKNOWLEDGMENTS}

This work was supported by the Methusalem Funding by the Flemish Goverment, the European Science Foundation (ESF), NES program, the IAP-P6/42 project "Quantum Effects in Clusters and Nanowires," GOA, and FWO projects.

*bramleo@ hotmail.com

${ }^{1}$ M. Stoneham, Nature Mater. 3, 3 (2004).

${ }^{2}$ H. Ye, N. Tumilty, M. Bevilacqua, S. Curat, M. Nesladek, B. Bazin, P. Bergonzo, and R. B. Jackman, J. Appl. Phys. 103, 054503 (2008).

${ }^{3}$ E. A. Ekimov, V. A. Sidorov, E. D. Bauer, N. N. Mel'nik, N. J. Curro, J. D. Thompson, and S. M. Stishov, Nature (London) 428, 542 (2004).

${ }^{4}$ K. Thonke, Semicond. Sci. Technol. 18, S20 (2003).

${ }^{5}$ T. Klein, P. Achatz, J. Kacmarcik, C. Marcenat, F. Gustafsson, J. Marcus, E. Bustarret, J. Pernot, F. Omnes, B. E. Sernelius, C. Persson, A. Ferreira da Silva, and C. Cytermann, Phys. Rev. B 75, 165313 (2007).

${ }^{6}$ W. Gajewski, P. Achatz, O. A. Williams, K. Haenen, E. Bustarret, M. Stutzmann, and J. A. Garrido, Phys. Rev. B 79, 045206 (2009).

${ }^{7}$ J. J. Mareš, P. Hubík, M. Nesládek, and J. Krištofik, Diamond Relat. Mater. 16, 921 (2007).

${ }^{8}$ Y. Takano, T. Takenouchi, S. Ishii, S. Ueda, T. Okutsu, I. Sakaguchi, H. Umezawa, H. Kawarada, and M. Tachiki, Diamond Relat. Mater. 16, 911 (2007).

${ }^{9}$ O. A. Williams, M. Nesladek, M. Daenen, S. Michaelson, A. Hoffman, E. Osawa, K. Haenen, and R. B. Jackman, Diamond Relat. Mater. 17, 1080 (2008).

${ }^{10}$ C. Wild, P. Koidl, W. Müller-Sebert, H. Walcher, R. Kohl, N. Herres, R. Locher, R. Samlenski, and R. Brenn, Diamond Relat. Mater. 2, 158 (1993); C. Wild, R. Kohl, N. Herres, W. Müller-

Sebert, and P. Koidl, ibid. 3, 373 (1994).

${ }^{11}$ B. Sacepe, C. Chapelier, C. Marcenat, J. Kačmarčik, T. Klein, M. Bernard, and E. Bustarret, Phys. Rev. Lett. 96, 097006 (2006).

${ }^{12}$ E. A. Ekimov, V. A. Sidorov, A. V. Zoteev, J. B. Lebed, J. D. Thompson, and S. M. Stishov, Sci. Technol. Adv. Mater. 9, 044210 (2008).

${ }^{13}$ I. B. Altfeder, J. J. Hu, A. A. Voevodin, and J. Krim, Phys. Rev. Lett. 102, 136104 (2009).

${ }^{14}$ A. D. Truscott, R. C. Dynes, and L. F. Schneemeyer, Phys. Rev. Lett. 83, 1014 (1999).

${ }^{15}$ M. Vinet, C. Chapelier, and F. Lefloch, Phys. Rev. B 63, 165420 (2001).

${ }^{16}$ W. Escoffier, C. Chapelier, N. Hadacek, and J.-C. Villégier, Phys. Rev. Lett. 93, 217005 (2004).

${ }^{17}$ Ya. V. Fominov and M. V. Feigel'man, Phys. Rev. B 63, 094518 (2001).

${ }^{18}$ O. A. Williams, O. Douhéret, M. Daenen, K. Haenen, E. Osawa, and M. Takahashi, Chem. Phys. Lett. 445, 255 (2007).

${ }^{19}$ K. K. Likharev, Dynamics of Josephson Junctions and Circuits (Gordon and Breach Science, France, 1984).

${ }^{20}$ I. Guillamón, H. Suderow, F. Guinea, and S. Vieira, Phys. Rev. B 77, 134505 (2008).

${ }^{21}$ G. Rubio-Bollinger, H. Suderow, and S. Vieira, Phys. Rev. Lett. 86, 5582 (2001).

${ }^{22}$ The STM and STS data were analyzed by using the WSxM 4.0 Develop 12.1-image browser software by I. Horcas, R. Fernández, J. M. Gómez-Rodríguez, J. Colchero, J. Gómez-Herrero, 
and A. M. Baro, Rev. Sci. Instrum. 78, 013705 (2007).

${ }^{23}$ C. A. M. dos Santos, C. J. V. Oliveira, M. S. da Luz, A. D. Bortolozo, M. J. R. Sandim, and A. J. S. Machado, Phys. Rev. B 74, 184526 (2006).

${ }^{24}$ B. L. Willems, G. Zhang, J. Vanacken, V. V. Moshchalkov, S. D. Janssens, O. A. Williams, K. Haenen, and P. Wagner, J. Appl. Phys. 106, 033711 (2009).

${ }^{25} \varnothing$. Fischer, M. Kugler, I. Maggio-Aprile, C. Berthod, and C. Renner, Rev. Mod. Phys. 79, 353 (2007).

${ }^{26}$ O. A. Williams and M. Nesládek, Phys. Status Solidi A 203, 3375 (2006).

${ }^{27}$ P. G. de Gennes, Rev. Mod. Phys. 36, 225 (1964).

${ }^{28}$ T. Nishizaki, Y. Takano, M. Nagao, T. Takenouchi, H. Kawarada, and N. Kobayashi, J. Phys. Chem. Solids 69, 3027 (2008).

${ }^{29}$ K. M. Lang, V. Madhavan, J. E. Hoffman, E. W. Hudson, H. Eisaki, S. Uchida, and J. C. Davis, Nature (London) 415, 412 (2002).

${ }^{30}$ J. E. Hoffman, K. McElroy, D.-H. Lee, K. M. Lang, H. Eisaki, S. Uchida, and J. C. Davis, Science 297, 1148 (2002).

${ }^{31}$ K. McElroy, R. W. Simmonds, J. E. Hoffman, D.-H. Lee, J. Orenstein, H. Eisaki, S. Uchida, and J. C. Davis, Nature (London) 422, 592 (2003).

${ }^{32}$ K.-W. Lee and W. E. Pickett, Phys. Rev. Lett. 93, 237003 (2004).

${ }^{33}$ T. Yokoya, T. Nakamura, T. Matsushita, T. Muro, Y. Takano, M. Nagao, T. Takenouchi, H. Kawarada, and T. Oguchi, Nature (London) 438, 647 (2005).
${ }^{34}$ M. Cardona, Sci. Technol. Adv. Mater. 7, S60 (2006).

${ }^{35}$ K. D. Usadel, Phys. Rev. Lett. 25, 507 (1970); W. Belzig, F. Wilhelm, C. Bruder, G. Schön, and A. D. Zaikin, Superlattices Microstruct. 25, 1251 (1999).

${ }^{36}$ The condition of zero derivative corresponds to an insulating boundary. It happens that the same set of equations also describe a periodic system of alternating $\mathrm{S}$ and $\mathrm{N}$ layers of respective thickness $2 L_{\mathrm{S}}$ and $2 L_{\mathrm{N}}$.

${ }^{37}$ J. J. Lin and J. P. Bird, J. Phys.: Condens. Matter 14, R501 (2002).

${ }^{38}$ P. Martìnez-Samper, H. Suderow, S. Vieira, J. P. Brison, N Luchier, P. Lejay, and P. C. Canfield, Phys. Rev. B 67, 014526 (2003).

${ }^{39}$ E. Bustarret, J. Kačmarčik, C. Marcenat, E. Gheeraert, C. Cytermann, J. Marcus, and T. Klein, Phys. Rev. Lett. 93, 237005 (2004).

${ }^{40}$ W. H. Li, C. C. Yang, F. C. Tsao, and K. C. Lee, Phys. Rev. B 68, 184507 (2003).

${ }^{41}$ A. M. Clogston, Phys. Rev. Lett. 9, 266 (1962).

${ }^{42}$ J. Von Delft and D. C. Ralph, Phys. Rep. 345, 61 (2001).

${ }^{43}$ In fact, highly dispersive bands have been showed to approach each other at the Fermi level in heavily boron-doped diamond (Ref. 33) with the values for their $k_{F}$ equal to $k_{F}^{A}=-1.5 \mathrm{~nm}^{-1}$, $k_{F}^{B}=1.1 \mathrm{~nm}^{-1}$, and $k_{F}^{C}=2.7 \mathrm{~nm}^{-1}$. For simplicity, the estimation of $H_{\text {orb }}$ has been done by using the free-electron approximation for the $k$ space of the boron-doped diamond. 